\title{
POLÍTICAS DE EVALUACIÓN ESTANDARIZADA Y GOBERNANZA "EMPRESARIAL" EN EDUCACIÓN
}

\author{
STANDARDIZED ASSESSMENT POLICIES AND "BUSINESS" \\ GOVERNANCE IN EDUCATION
}

\author{
Enrique-Javier Díez-Gutiérrez
}

\begin{abstract}
This article analyses closely the policies of standardized evaluation that continue to be implemented throughout the world and which represent one of the most incisive mechanisms of the new paradigm of "neo-liberal governance" in education. It investigates how this evaluation model demands the implementation of new managerial "business" style techniques in educational institutions and it is supported by rhetoric that proclaims the necessity of decentralization and of educational autonomy for schools. However, this discourse does not refer to pedagogical autonomy, but to financial autonomy - decentralization without resources. In this context, the socalled "New Public Management" comes to the fore as a mechanism of governance for public services, applied to education. The article explores how these stratagems operate, what they "make one do" and how they become tools of social control since they require the active participation of those involved and impose a rationale oriented to "self-governance" under the pressure of competition, in accordance with the principle of calculating maximum individual interest. Finally, in a proactive sense, this article raises the need for a different approach to educational evaluation and management that is both possible and necessary in a democratic and inclusive model.
\end{abstract}

Key words: Standardized evaluation policies, Neoliberal governance, New Public Management, Educational autonomy, Business management.

\section{RESUMEN}

Este artículo analiza las políticas de evaluación estandarizadas que se siguen implantando en todo el mundo, como uno de los mecanismos más incisivos del nuevo paradigma de "gobernanza neoliberal" educativa. Indagando cómo este modelo de evaluación está demandando una nueva gestión al estilo "empresarial" de los centros educativos, acompañada por un discurso que proclama la necesaria descentralización y autonomía educativa de los centros. Pero no se refiere a una autonomía pedagógica, sino de financiación. Una descentralización, pero sin recursos. En este contexto, resurge la denominada "Nueva Gestión Pública", como mecanismo de gobernanza de los servicios públicos, aplicada a la educación. En el texto se explora cómo operan estos dispositivos, que "hacen hacer" y que se convierten en herramientas de control social. Puesto que requieren la participación activa de los involucrados e imponen una "racionalidad" orientada a "gobernarse" bajo la presión de la competición, de acuerdo con el principio de cálculo del máximo interés individual. Finalmente, en sentido propositivo, este artículo plantea la necesidad de otro enfoque de la evaluación y la gestión educativa posible y necesario, desde un modelo democrático e inclusivo.

Palabras clave: Políticas de evaluación estandarizadas, Gobernanza neoliberal, Nueva Gestión Pública, Autonomía educativa, Gestión empresarial. 
Fecha de recepción: 26 de marzo de 2020.

Fecha de aceptación: 15 de julio de 2020. 


\section{INTRODUCCIÓN}

La evaluación estandarizada se está configurando como uno de los mecanismos más incisivos del nuevo paradigma de "gobernanza neoliberal" educativa en todo el mundo. Este enfoque de la evaluación permite comprender la operatividad de los dispositivos neoliberales que "hacen hacer" (Boltanski, 2009), sin recurrir principalmente a la obediencia y a la coerción explícitas, sino disponiendo las condiciones materiales y discursivas para acciones y comportamientos en la que quienes participan se comprometen "libremente", aunque sea de manera ambivalente, y que se constituyen como procesos de normalización de prácticas vinculadas a la lógica neoliberal con efectos de subjetivación y asunción de la propia dominación (Amigot \& Martínez, 2015).

Por eso, quizá la pregunta crucial en evaluación sea el para qué se evalúa. Es decir, la finalidad de la misma. Lo que tradicionalmente ha perseguido la evaluación educativa es aportar datos, hechos y elementos de juicio que posibiliten la toma de decisiones racionalmente fundamentadas para mejorar y hacer del proceso de enseñanza-aprendizaje un factor de cambio social desde una perspectiva de justicia y solidaridad (Perrenoud, 1990).

Pero no parece que éste sea el rumbo que se haya establecido en los programas de evaluación estandarizada, que se están implantando a nivel internacional. Más bien, se trata de evaluar para controlar, para exigir un incremento del rendimiento "útil” y medible y de la extensión de la lógica competitiva en educación. Centrados en el control formal de los centros y la exhibición de sus resultados en rankings, presionan a los centros para competir entre ellos, en vez de colaborar, con el fin de escalar posiciones y así ser más "demandados" por las familias-clientes, que se acaban autoconcibiendo como tales en un este "mercado educativo" de oferta y demanda.

Esta dinámica está transformando la educación en una inversión personal, donde los "clientesinversores" demandan resultados constatables de su inversión, donde los centros priorizan aquellos aspectos de moda que más pueden atraer nuevos clientes (bilingüismo, mindfulness, programas de emprendimiento, marketing publicitario, robótica o gamificación, etc.) y donde se comienza a ver al alumnado con mayores dificultades como una dificultad añadida para mantenerse o escalar en los rankings.

Este modelo de evaluación está demandando una nueva gestión al estilo "empresarial" de los centros educativos, que refuerza un control formal y un modelo de dirección-gerencia unipersonal, supuestamente técnica y profesional, que sea eficiente en conseguir los resultados formales demandados, de cara a figurar en el ranking de excelencia. Es lo que se ha denominado "Nueva Gestión Pública", aplicada a la educación: gestionar los centros educativos como si fueran empresas "eficientes" y rentables.

\section{LA EVALUACIÓN COMO RENDICIÓN DE CUENTAS}

Las políticas de rendición de cuentas han surgido ligadas a esta Nueva Gestión Pública como mecanismo de gobernanza de los servicios públicos mediante agencias evaluadoras. El modelo de rendición de cuentas más extendido ha adquirido un enfoque gerencial centrado en los resultados. En el campo de la educación, se ha convertido en el modelo de reforma educativa global, a pesar de que no hay evidencias de que tengan efectos positivos (sino más bien al contrario, especialmente respecto al incremento de los niveles de aprendizaje, la mejora de los procesos educativos o la reducción de las desigualdades educativas), centrándose en los resultados de aprendizaje de los estudiantes a través de instrumentos de evaluación estandarizada (Hamilton \& otros, 2002; Hewitt, 2015; Lane, 2014).

Mediante estas evaluaciones estandarizadas externas y la distribución de incentivos o sanciones, en función de los resultados obtenidos en dichas evaluaciones, se pretende asegurar el control y presionar a escuelas y profesorado para que cumplan y se ajusten a estándares y resultados exigidos externamente. Modifican así las dinámicas de autorregulación colaborativas que habían prevalecido 
en la profesión docente y en las comunidades educativas, en las que la evaluación es asumida como una responsabilidad compartida entre las comunidades educativas (Verger \& Parcerisa 2017).

La dinámica introducida por la actual carrera competitiva económica internacional, donde la educación deviene una pieza considerada clave para aumentarla, ha convertido la adopción de estos sistemas de evaluación estandarizados, impulsados por la Organización para la Cooperación y el Desarrollo Económico (OCDE), como PISA, TIMSS, PIRLS u otras similares, en una clave estratégica en esta "carrera educativa" global para medir la mejora de los resultados de aprendizaje. Aunque realmente no sirven para ayudar a mejorar la enseñanza en el aula y el funcionamiento de los centros educativos (Carabaña, 2015).

A pesar de ello, los resultados arrojados por este tipo de pruebas se han convertido en el indicador internacional que genera rankings comparativos entre centros y naciones debido a que se trata de un indicador concreto y fácilmente comparable, que genera titulares de prensa. Otros componentes del proceso educativo, más sensibles al contexto o más difíciles de reflejar en pruebas de evaluación estandarizada, quedan fuera de esta evaluación reducida a "rendición de cuentas", a pesar de que son los que realmente reflejan dimensiones esenciales del proceso educativo.

Y, como dicen Verger \& Parcerisa (2017) "la implementación de sistemas de rendición de cuentas permite a los gobiernos transmitir a los votantes -y a la ciudadanía en general- el mensaje de que están trabajando arduamente para el cambio educativo, y para la mejora de los resultados de aprendizaje y de las oportunidades futuras de los niños y niñas. Al mismo tiempo, las reformas de rendición de cuentas tienden a implicar un bajo riesgo político ya que, en gran medida, la presión de la reforma recae en las escuelas y en los maestros, y no tanto en el gobierno, y son más baratas y rápidas que reformas alternativas". Sin olvidar que las empresas que venden servicios relacionados con la medición de resultados de aprendizaje, para la implementación y/o el procesamiento de los datos de pruebas externas, también son agentes activos en la propagación de reformas de rendición de cuentas. Según la OCDE, el hecho de que "la evaluación estandarizada de los estudiantes se convierta en una industria más que rentable" significa que "las empresas tienen fuertes incentivos para presionar por la expansión de la evaluación estandarizada de los estudiantes" (OCDE, 2013, 51). Añadiendo que organismos internacionales como la OCDE, el Banco Mundial o la UNESCO están intentando crear un consenso alrededor de la deseabilidad de adoptar sistemas de rendimiento de cuentas en el campo educativo que promuevan dinámicas de mercado y elección escolar.

\subsection{El GIRO NEOLIBERAL EN LA EVALUACIÓN}

En los últimos años el auge del modelo neoliberal en educación, presentado como "profesional y tecnocrático", centrado en pruebas estandarizadas, ha sido importado acríticamente en buena parte de los países industrializados, por presiones de organismos internacionales de carácter económico, como el FMI, el Banco Mundial o la OCDE, justo en momentos en que dichas pruebas son debatidas, cuestionadas y refutadas por especialistas y expertas en educación (Andrews \& al., 2014; Carabaña, 2015; Gentili, 2017; Fernández, 2018; Gómez, 2018; Merchán, 2012; Mons, 2009; Ravitch, 2013; Sharon \& Berliner, 2007; Stobart, 2010; Wrigley, 2007).

El informe PISA, denominado así por sus siglas en inglés (Programme for International Student Assessment) puede ser un buen exponente, que asume buena parte de este enfoque del modelo neoliberal de evaluación. Este informe refleja la valoración y comparación internacional del alumnado de 15 años mediante la realización de pruebas estandarizadas o exámenes en las áreas de Lectura, Matemáticas y Ciencias. Aunque ya está en marcha un 'baby PISA', estudio piloto internacional en Inglaterra y EEUU, en el que niños y niñas de cinco años se verán sometidos a 'tests' para evaluar su comportamiento social, empatía, memoria y autorregulación, así como sus habilidades en lenguaje, lectoescritura y aritmética (Morton, 2017).

El impacto que ha adquirido este informe tiene que ver con el giro económico neoliberal que se está produciendo en la concepción de la educación a nivel mundial. 
Como denuncian intelectuales del Consejo Latinoamericano de Ciencias Sociales (CLACSO), PISA y los programas de evaluación estandarizada son un inmenso dispositivo de control que aspira a imponer una perspectiva educativa que aleja del reconocimiento de la educación como un derecho y aproxima a su interpretación como un bien de consumo. Una concepción de la educación entendida como una ventaja competitiva, en la que cada individuo invierte de cara a su inserción más exitosa en el futuro laboral (Feldfeber \& Assael, 2014).

Esta filosofía neoliberal ha irrumpido con fuerza en el escenario del sistema educativo desde finales de los años 80. Proporcionar al mercado trabajadoras y trabajadores adaptados a las exigencias de la producción moderna, se ha convertido, con mucho, en la tarea primordial y la más importante de las funciones atribuidas a la enseñanza, en este "giro neoliberal". De aquí que los modelos de evaluación adoptados con estos programas de evaluación estandarizada tratan de comprobar la adecuación de los futuros trabajadores y trabajadoras a las exigencias del mercado.

Las inversiones en el sistema educativo comienzan a ser pensadas de acuerdo con las exigencias del mercado, y su rentabilidad debe ser evaluada conforme a ello. La persona trabajadora "flexible" y "polivalente" constituye así la referencia del nuevo ideal pedagógico. El papel público de la educación como campo de entrenamiento para la democracia y para la ciudadanía democrática se ha pasado a considerar como un despilfarro del gasto público, siendo reemplazado por el punto de vista que la empresa privada tiene de la función de la enseñanza: un campo de entrenamiento para atender las necesidades de las empresas.

Se emprende así una nueva cruzada de reconceptualización del discurso sobre las prioridades de la educación y una nueva retórica sobre los nuevos "desafíos" de nuestra época y los escenarios futuros, siempre con la finalidad de ajustar la educación a las demandas del mercado laboral. Como si los seres humanos se pensaran y definieran únicamente como trabajadores y trabajadoras de la maquinaria laboral. De esta forma se está produciendo una auténtica mutación en la naturaleza y fines de la educación que, de formar ciudadanos y ciudadanas provistos de valores, saberes críticos y capacidades, pasa a subordinarse completamente a la producción de "recursos humanos" para el sistema productivo.

A partir de la década de 1970, se trata de pensar el sistema educativo en términos de salidas profesionales y evaluarlo en función de ello. La problemática de la inserción laboral prevalece sobre la aspiración a la integración social y política de los futuros ciudadanos y ciudadanas. La profesionalización ya no es una finalidad entre otras del tramo final de la escolarización, sino que tiende a convertirse en la principal línea directriz de todas las reformas y las políticas de evaluación educativa.

En este modelo neoliberal, la función social asignada a la educación se centra en su apoyo al crecimiento económico, su aportación a la competitividad empresarial de las industrias, la formación para el trabajo y la capacitación para el desarrollo tecnológico. Estas funciones económicas priman sobre la función de socializar para participar activamente en una ciudadanía consciente y comprometida, transmitir la cultura y desarrollar la personalidad.

Por eso no es de extrañar que estas pruebas estandarizadas se lleven a cabo por la Organización para la Cooperación y el Desarrollo Económicos (OCDE), organismo internacional creado para "maximizar el crecimiento económico" de los países que lo integran, con la intención, afirman, de "proporcionar a los gobiernos datos relevantes y fiables que les permitan tomar decisiones en materia de política educativa".

La OCDE es un organismo económico, con un sesgo muy caracterizado a favor del papel económico que la educación tiene que jugar en el modelo neoliberal de sociedad que potencia. Es a esta institución, con una clara orientación economicista, a la que nuestros representantes políticos han decidido encomendar ser el árbitro global de los medios y fines de la educación y determinar lo que los estudiantes deben saber y las escuelas enseñar, asumiendo el poder de configurar la política educativa, sin debate acerca de la necesidad o de las limitaciones de las metas de esta institución económica. 
Una orientación economicista y mercantil que no centra la evaluación en objetivos educativos realmente importantes pero que son menos susceptibles o imposibles de ser medidos, pues tiene que cuantificar, reduciendo de esta forma la imaginación colectiva en torno a lo que es o debería ser la educación.

\subsection{PISA Y LA EVALUACIÓN ESTANDARIZADA}

El éxito mediático y político del informe PISA se debe, sobre todo, a que los resultados se publican en forma de ranking o clasificación mundial y un sector político los utilizan para justificar sus reformas educativas y atacar las de sus contrarios, argumentado la incapacidad de éstos para hacer brillar a la nación en el palmarés de la excelencia de los rankings internacionales (tradicional método escolar "jesuítico" de competición escolar, aunque con el lenguaje renovado de la excelencia académica y las competencias).

$\mathrm{Al}$ calor de este giro neoliberal, se ha popularizado una "neolengua" tecno-educativa, con sabor a presunta modernidad importada del mundo empresarial, en el que se propone "medir" determinadas competencias y obtener así resultados "comparables" que se ofrecen en forma de clasificación o ranking, como si de una liga de futbol se tratara. Los "clientes" de este nuevo "supermercado educativo" podrán así comparar y seleccionar el "producto educativo" de ese ranking que mejores ventajas competitivas les ofrezca, de cara al futuro laboral de su prole. Ya no se plantea, por tanto, la educación como un derecho de todos los niños y las niñas, que se ha de garantizar en igualdad de condiciones desde la equidad y la justicia social, sino como una inversión personal en la que cada familia o individuo compite por conseguir la mejor rentabilidad posible de dicha inversión.

En 2007, un grupo de investigadoras e investigadores (Bodin, 2007; Brinek \& Hopmann, 2007) analizó de forma pormenorizada la metodología de PISA y publicó sus conclusiones en el libro PIS $A$ according to PIS A. Además de asegurar que los rankings que comparan los resultados entre países "están basados en tantos puntos débiles que deben ser abandonados de inmediato", apuntaron que los productos asociados a PISA, como los análisis sobre cómo deben ser las buenas escuelas o las diferencias entre los distintos sistemas educativos, "van mucho más allá de lo que permite una aproximación cauta a estos datos. Son en su mayoría pura especulación”.

Lo cierto es que este programa de evaluación estandarizada ${ }^{1}$ está muy cuestionado respecto a su valor para mejorar la enseñanza en las aulas y el funcionamiento de las escuelas (Carabaña, 2015). Como explica este experto, mide capacidades que dependen tanto de lo que sucede en la escuela como de la experiencia acumulada en la vida del alumnado y que la prueba no puede medir. Incluso en sus propios textos PISA reconoce que "si un pais puntúa más que otro no se puede inferir que sus escuelas sean más efectivas, pues el aprendizaje comienza antes de la escuela y tiene lugar en una diversidad de contextos institucionales y extraescolares".

Además de que PISA sólo evalúa los conocimientos y competencias de alumnado de 15 años en matemáticas, ciencia y compresión lectora, pero ni realiza un seguimiento longitudinal de la evolución de los estudiantes, ni se para a analizar qué saben de otras materias o habilidades que van de la historia a la filosofía, a la creatividad o al arte. No solo estandariza, encubriendo la pretensión de hegemonizar, a través de su medición, los valores de una determinada sociedad, sino que convierte en secundarios y marginales esos "otros" saberes (filosofía, enseñanzas artísticas o musicales, etc.) que "distraen" de que se tenga un buen resultado en dichas pruebas PISA.

En este sentido, muchos expertos y expertas se preguntan si lo que mide PISA es la creatividad o la adaptación al modelo neoliberal de nuestros jóvenes (Gómez, 2018). Si lo que se pretende es presentar la estandarización bajo los ropajes de la objetividad, cuando lo cierto es que en el fondo

\footnotetext{
1 Aunque el análisis se centra en PISA por su repercusión mundial, las investigaciones demuestran que es aplicable a otros programas de evaluación estandarizada como el SIMCE que, al igual que PISA, "no tiene utilidad pedagógica en el aula ni en los establecimientos" (Stuardo-Concha, 2018).
} 
encubre la pretensión de hegemonizar, a través de su medición, los valores de una determinada sociedad (Vaca, 2005). ¿Por qué no observar el desarrollo de las emociones, las capacidades de interrelación, la creatividad de los jóvenes en su contexto cultural y social?, se preguntan. Quizá por ello PISA y PIRLS, prueba menos conocida y centrada en lengua, ya han comenzado a incluir algún indicador relacionado con lo emocional (OECD, 2017; Mullis \& al., 2017), preguntando al alumnado sobre su nivel de satisfacción general y con el propio desempeño, su sentimiento de pertenencia en la escuela o su ansiedad ante el trabajo escolar.

También se cuestionan sus deficiencias y limitaciones metodológicas referidas a una lógica incoherente, toma de muestras opacas, diseño evaluativo inestable, instrumentos de medición de validez cuestionables, el uso oportunista de las puntuaciones transformadas por la normalización, la confianza reverencial en la significación estadística, la ausencia de estadísticas sustantivamente importantes centradas en las magnitudes de los efectos, una presentación problemática de los hallazgos e implicaciones cuestionables extraídas de los resultados para las prácticas y las legislaciones educativas (Fernández, 2016).

Otras críticas se centran en la aplicación de las mismas pruebas estandarizadas a alumnado de 15 años, independientemente de las diferencias significativas en relación con su proceso educativo y a su escolaridad, e indistintamente del curso en el que se encuentren y de los contenidos que hayan estudiado (sobre todo en el caso de que haya una alta tasa de repetición entre el alumnado de 15 años, como es el caso de España).

También el hecho de que la obsesión de preparase para este tipo de exámenes estandarizados da un peso excesivo a las habilidades para seguir instrucciones y procedimientos, en desmedro de verdaderas estrategias de innovación o creatividad. Tampoco tiene en cuenta el efecto que los docentes tienen sobre las habilidades no cognitivas, tan importantes o más que las propias habilidades cognitivas (Kirabo, 2012; 2018). Ni cómo esta presión por la rendición de cuentas está afectando al profesorado (Dizon-Ross, 2020; Feng et al., 2018; Gjefsen \& Gunnes, 2016).

Tampoco PISA tiene en cuenta dónde está la escuela, de dónde se partía cuando se inició el proceso educativo o que transformaciones se han logrado con la intervención pedagógica.

Más críticas apuntan a la concepción homogeneizadora de las pruebas sin tener en cuenta el contexto socioeconómico y cultural de cada país y región, cuestionando los sesgos socioculturales de los ítems de las pruebas. Como si solo existiera un mundo, una única cultura y una única forma de insertarse productivamente en este mundo (Leal \& Perez, 2014).

También se cuestiona para qué seguir haciendo estas pruebas tan costosas y que suponen un desembolso público muy considerable, en un contexto de recortes continuados en lo educativo, puesto que reiteran periódicamente lo que ya de hecho sabemos. Los resultados de estas pruebas estandarizadas nada aportan que no sepamos sobre el funcionamiento de nuestros sistemas escolares (Carabaña, 2016).

Porque efectivamente, y este es un aspecto que no debemos olvidar, las multinacionales del mundo editorial y relacionadas con la educación privada están desembarcando en este suculento negocio que comporta muchos millones de beneficios. Pearson, la corporación del negocio educativo más grande del mundo, se hizo con el contrato para la gestión de los exámenes de PISA $^{2}$ y de la plataforma digital que los sustenta. Contrato pagado con dinero público por los ministerios de Educación de los países participantes. Es decir, esta multinacional no solo redacta los exámenes, también los corrige y aporta las herramientas informáticas para analizarlos. De esta forma, Pearson utiliza PISA como cabeza de puente para manejar los hilos de la educación mundial. Al menos le sitúa en una posición muy ventajosa a la hora de vender sus servicios a aquellos gobiernos que quieran conseguir mejorar sus resultados en las próximas evaluaciones.

Para Diane Ravitch (2013), experta educativa de la Universidad de Nueva York, estamos ante "la irrupción del big data en las escuelas", en donde grandes fondos de capital riesgo ya están entrando en

${ }^{2}$ https://uk.pearson.com/about-us/news-and-policy/news/2011/09/pearson-to-develop-frameworks-for-oecda--s-pisa-student-assessme.html 
este mercado, invirtiendo en compañías digitales que se dedican a desarrollar apps, diseñar y evaluar exámenes y vender datos de estudiantes para aumentar sus márgenes de beneficio aún más.

Como analiza Gentili (2017), no es coincidencia que PISA exista en un contexto en el que los grandes monopolios de la producción de los libros de texto han crecido exponencialmente. Un sistema como el de PISA genera enormes beneficios económicos al que produce las herramientas para examinar de forma estandarizada en todo el mundo y materiales didácticos estandarizados para tener "éxito" en esas pruebas. Además de darle un control inmenso sobre lo que los niños y niñas de todo el mundo tienen que estudiar y el profesorado enseñar.

El problema colateral de este fenómeno es que los países están emprendiendo una loca y desenfrenada carrera por estar en los puestos superiores del ranking, pues quien pierde el tren de esa supuesta "excelencia" acaba descarrilando. Lo cual implica que se exige al profesorado que se centre en buscar la forma de obtener resultados, dedicando el tiempo a preparar lo que le piden en las pruebas o a conseguir aquello que les sitúe en la cúspide del ranking (Deming et al., 2016). Pero, a su vez, el alumnado con dificultades y diversidad se convierte en un estorbo y una posibilidad de que el centro baje puestos en el ranking si lo admite o dedica mucho tiempo a su atención. Por lo que se produce un efecto de inversión en la práctica educativa profundamente corrosivo: ya no se piensa qué puede hacer el centro por el alumno o alumna, sino qué pueden hacer ellos porque el centro mejore su posición en los resultados del ranking de competencias.

La importancia desmedida que se le está concediendo socialmente a este tipo de evaluaciones estandarizadas, por su impacto mediático, puede significar un cambio crucial en los objetivos de la escuela. Con la llegada de los informes PISA, se empieza a poner el acento en medir el rendimiento del estudiante más que en atender las necesidades del mismo.

En el año 2014, 83 expertos internacionales de reconocido prestigio en educación, enviaron una carta al director del programa PISA (Andrews \& al., 2014), expresando su preocupación por que, como resultado de PISA, muchos países están siendo presionados para introducir reformas educativas cortoplacistas con el fin de escalar posiciones en dicho ranking y presentar resultados ante los medios, asegurando que la educación del país mejora si sube en esa escala, sin entender que los cambios y mejoras de un sistema educativo necesitan plazos y tiempos prolongados.

Lo que realmente está introduciendo este organismo económico internacional, la OCDE, con estas evaluaciones estandarizadas, es un mecanismo de control "a distancia" de los gobiernos nacionales (Rose, O’Malley \& Valverde, 2006) y una forma de gobernar a través de la estadística y los números (Grek, 2009), que genera una competencia permanente entre los propios centros educativos y entre las regiones y los países por figurar en la parte alta de los rankings de PISA.

Los especialistas (Carabaña, 2015; Gentili, 2017; Ravitch, 2013) apuntan que el régimen de PISA, con su ciclo continuo de medición global, está haciendo daño al alumnado y empobreciendo la educación, aumentando aún más el ya alto nivel de estrés en las escuelas, con una presión constante por el rendimiento. Alertando de que esta dinámica supone un riesgo real de matar el placer de aprender, transformando el deseo de aprender en afán de aprobar.

En definitiva, los efectos negativos más evidentes que se han observado (Merchán, 2012; Mons, 2009; Sharon \& Berliner, 2007; Stobart, 2010; Wrigley, 2007), respecto a las evaluaciones estandarizadas, son:

a) La presión sobre los niños y niñas para que tengan resultados acomodados a las pruebas.

b) El estrés del control continuo y permanente, como si solo se pudiera aprender mediante la presión y el miedo al examen, olvidando la curiosidad y la motivación por conocer.

c) La deslegitimación de la función docente y la desconfianza hacia la evaluación del profesorado.

d) La selección de aquellos "clientes" que son buenos para no descender en el ranking;

e) La degradación de contenidos: se acaba estudiando lo que se somete a examen, y se centra el tiempo y los esfuerzos docentes en preparar al alumnado para superar pruebas y exámenes. 
f) El control sobre el trabajo docente y la pérdida de su autonomía educativa, al convertirse el profesorado en "preparadores de pruebas", sufriendo así un control directo sobre su trabajo y sobre lo que debe enseñar.

g) El coste económico para el sistema, tan alto como inútil, por la realización de las múltiples pruebas externas todos los años. Y el coste añadido para las familias del alumnado que haya suspendido cuando tengan que pagar academias para repetir la reválida.

h) La injusticia para el alumnado, que ha de jugarse en una prueba externa varios años de escolarización, frente a la función de la evaluación como instrumento de mejora de la educación, respetando la diversidad y los ritmos de aprendizaje.

i) El gran negocio que supone transferir millones de euros a grandes empresas como Pearson, McGraw-Hill y Educational Testing ${ }^{3}$ (Lingard \& al., 2017).

j) La publicidad de estas pruebas en rankings, configurando listados de centros ordenados en función de las puntuaciones obtenidas por el alumnado, que convierte la evaluación en un mecanismo de competición entre centros y no de cooperación; trasladando la responsabilidad de estos resultados a los centros y al profesorado, pues a los docentes es a quienes primero se responsabiliza de los malos resultados, sin tener en cuenta el conjunto de factores asociados a los rendimientos escolares.

\section{Nueva Gestión PÚblica (NGP) EN LA EDUCACión}

Como decíamos anteriormente, este modelo de evaluación estandarizada está vinculado a una gestión al estilo "empresarial" de los centros educativos, a lo que se ha denominado "Nueva Gestión Pública", aplicada a la educación: gestionar los centros educativos como si fueran empresas "eficientes" y rentables. Este nuevo paradigma de "gobernanza neoliberal", opera con dispositivos que "hacen hacer", como la evaluación estandarizada.

Michel Foucault (1975), señalaba que las sociedades occidentales actuales han abandonado el modelo disciplinario y, en contraste, han adoptado herramientas de control social que requieren la participación activa de los involucrados (Foucault, 1975; Hardt \& Negri, 2002; Guattari \& Rolnik, 2006). Esta ideología neoliberal se ha convertido así en una "racionalidad" que se orienta a la conducción de las conductas y las prácticas (Foucault, 2004; Ong, 2007) mediante procesos de subjetivación que pretenden imponer la competencia y hacer de ella una forma general de la sociedad, que guiará todas las relaciones humanas (Laval \& Dardot, 2013). Ha sido preciso para ello pensar e instalar, "mediante una estrategia sin estrategias", las formas de control y organización del proceso educativo que orienten a "gobernarse" bajo la presión de la competición, de acuerdo con los principios del cálculo del máximo interés individual.

Este modelo convierte a las personas y a las familias en "consumidoras" y "clientes" del sector educativo, que buscan aprovechar y maximizar sus oportunidades, generando, con su "demanda", competencia entre los establecimientos escolares, con el fin de que se esfuercen por alcanzar un alto puesto en los rankings y tengan, por tanto, más demanda, diseñando una gestión por rendimientos y objetivos, llevando incluso al profesorado a competir entre ellos. La competencia se convierte así en una forma de interiorización de las exigencias de rentabilidad, a la vez que se introduce una presión disciplinaria en la intensificación del trabajo, el acortamiento de los plazos, la individualización de los salarios y la precarización de las condiciones laborales del profesorado, reduciendo todas las formas colectivas de solidaridad en las comunidades educativas.

Esta tecnología de control "autodisciplinario" se acompaña simultáneamente de la expansión de toda una "tecnología evaluativa", entendida como medida del rendimiento y eficacia, la accountability, evaluación basada en los resultados medibles. Puesto que cuanto más "libre" se es de elegir en el mercado, más se necesita conocer la "calidad" de los productos que nos ofrecen, para elegir con

\footnotetext{
${ }^{3}$ En España el Gobierno destinó 14,5 millones en los presupuestos generales del 2016 a reválidas y evaluaciones finales solo en Primaria.
} 
"eficacia", a fin de aumentar las posibles ganancias individuales de nuestra inversión a futuro y competir con más probabilidades de éxito en la jungla de la competencia de todos contra todos en la sociedad.

Es este el enfoque de gubernamentalidad, de "gobierno-por-la-mente" que denunciaba Foucault (1975). Ya no requiere imposiciones externas, puesto que, efectivamente, invita a someterse por sí mismas a las personas, las comunidades educativas y los centros, que aprenden así a 'gobernarse' siguiendo ciertas normas y creencias, establecidas a distancia, que se van arraigando profundamente.

El nuevo sistema de gestión opera a través de estrategias de "soft power", mediante elementos indirectos de gobierno: el "gobierno a distancia", que encuadra los resultados exigidos y por tanto las prioridades educativas, a través de mecanismos como las evaluaciones estandarizadas, la rendición de cuentas o los rankings; así como la "libertad-responsabilidad" referida a la proclamada autonomía económica y financiera de los centros (no pedagógica), centrando así la responsabilidad de los resultados en los centros y las comunidades que han de ser "penalizadas" y castigadas si no los alcanzan, porque suya es la responsabilidad (Collet \& Tort, 2016).

Todas estas tecnologías de control y gestión sirven para "gobernar sin gobierno". Se presentan como elementos de eficiencia técnica, neutrales, profesionales y asépticos, en vez de como opciones ideológicas y políticas (Friz, 2016). Lo que importa en esta nueva gestión educativa de corte neoliberal, no es la legitimidad de partida, basada en valores y principios, sino la legitimidad de salida, basada en los resultados obtenidos (Ball, 2016, 29).

Eficacia, eficiencia, mínimo costo, corto plazo, competitividad, rendición de cuentas, lógica de beneficios, comparación de resultados, etc., son la nueva "lógica" que va penetrando, siendo vivida y practicada, compartida y proclamada de manera creciente, como la nueva "normalidad" (Collet \& Tort, 2016) y legitimadas como "evidencias científicas" a través de informes, estudios e investigaciones financiadas por los organismos y entidades (OCDE, FM, BM, FMI, etc.), defensoras del modelo neoliberal (Collet \& Tort, 2016, 131). Quien no los practica queda excluido o es tratado como "disidente"; quien los practica y no obtiene "éxito" debe asumir su responsabilidad y culpabilidad.

Políticas "interiores" que construyen y constituyen un tipo de subjetividad y de identidad acorde con los principios neoliberales, (re)socializando en principios, prácticas y valores neoliberales a personas y comunidades.

\subsection{MCDANALIZACIÓN "PROFESIONAL" DE LA EsCUELA}

Esta NGP, y la evaluación estandarizada vinculada a ella, requiere una organización de los centros educativos al estilo neoliberal: como si fueran una empresa privada, cuya forma de gestión se presenta como modelo de excelencia, en el paradigma neoliberal. Ritzer (1993) y Gentili (1997) denominan a este proceso Mcdonalización, la gestión de la escuela al estilo McDonald's.

Mcdonalizar la escuela, analiza Laval (2004), supone pensarla como una institución que debe adaptarse rápida y eficientemente a los estímulos o señales que emite un mercado altamente competitivo al que debe servir en la preparación de trabajadores y trabajadoras eficaces. Es evidente que la educación debe tener conexión con el mercado de trabajo; lo cuestionable es que parezca que sólo en función de ello se establezcan sus metas y se evalúe su impacto, como si lo auténticamente decisivo de la escuela fuera preparar la mano de obra de los procesos productivos (San Martín, 2006).

Se convierte así el proceso educativo en una labor meramente técnica. Se le cercena y se niega el eminente carácter social y político del acto educativo que implica conflictos de intereses, valores e ideales. Esta mutilación no admite que la comunidad educativa, protagonista del proceso educativo, se formule preguntas como "¿qué contenidos enseñar, para qué enseñarlos, a favor de qué o de quién enseñarlos?, ¿quién elige los contenidos y cómo se enseñan?, ¿cuál es el papel del profesorado y de las familias y del alumnado? ..." 
No entiende que la comunidad participe activamente en la escuela, ni que se organice democráticamente la misma.

La imitación del mundo de la empresa privada tiene como justificación la búsqueda de eficacia. La "escuela eficaz" debe regirse por la reducción y el control de los gastos educativos, que se ha vuelto una prioridad con el vacuo lema de: "hacer más con menos". De esta forma se justifica que los problemas no son de "inputs", es decir, no hay realmente problema de falta de recursos, sino de "outputs", de resultados que han de ser obtenidos de una forma eficaz, con menos recursos. Si ya no se pueden aumentar los recursos a causa del recorte de los gastos públicos y la reducción de los impuestos a las grandes fortunas o su desvío para rescatar a los bancos y financieras, el esfuerzo prioritario debe dirigirse hacia la administración "racional" de los sistemas escolares pasando, de las formas de organización democrática y participativa, con el concurso y la implicación de la comunidad educativa, a las formas de organización fundadas en el "gerencialismo" al estilo de las plantas industriales de fabricación. Se pone en marcha un verdadero culto a la eficacia y al rendimiento.

Para ello se necesitan, lógicamente, gestores y gestoras eficientes, cada vez más alejados de todo control democrático. Por eso se cambia el paradigma de entendimiento de la organización educativa, desviando el protagonismo de la comunidad educativa a los directores y directoras "eficaces", profesionalizando su función y orientándola hacia un modelo de gerencia, de gestión económica, empresarial y de recursos humanos. La participación es sustituida por la organización jerárquica y piramidal (Bolívar, López \& Murillo, 2013; González, 2017). Para ello es necesario poner al frente de las instituciones educativas a verdaderos directores-gestores encargados de aplicar eficazmente las políticas anunciadas como "modernización", decididas desde arriba y capaces de disciplinar y controlar a los docentes y al alumnado en la base. Es decir, un modelo basado en "la mucha vigilancia y la poca confianza", que cercena radicalmente la autonomía pedagógica, vendiéndose una "autonomía", que es realmente financiera y que lo que encubre es la falta de financiación y los recortes a la educación.

Este modelo jerárquico de organización genera un clima desmotivador, burocrático y autoritario, que destruye la confianza y la cooperación colectiva, justamente lo contrario que recomiendan todas las investigaciones sobre liderazgo y organización educativa (Bolivar, 2010, 2013; Bolívar, López \& Murillo, 2013; García, 2016; González, 2017; Navarro, Guzmán \& Guaderrama, 2016; Sebastián et al., 2016; Vargas, 2017).

En definitiva, la NGP pone en marcha dinámicas de cuasi-mercado que redefinen los aprendizajes desde un marco de rentabilidad, y también erosionan e incluso destruyen las relaciones colegiadas y colaborativas, el apoyo mutuo y el diálogo entre la comunidad educativa (Ball, 2003a, 2003b), elementos que, según todas las evidencias de la literatura científica y pedagógica, promueven un entorno que favorece tanto el propio desarrollo profesional del profesorado como el aprendizaje de sus estudiantes (Moreno-Hidalgo \& Manso, 2017; Verger, Bonal \& Zancajo, 2016), generando buen clima y cooperación entre el profesorado y la comunidad educativa, y equipos docentes y organizaciones que aprenden y pueden mejorar, así como sostener tales mejoras a lo largo del tiempo.

\subsection{LA TRAMPA DE LA “AUTONOMÍA” GERENCIAL}

Este proceso viene acompañado por un discurso que proclama la necesaria descentralización y autonomía educativa de los centros (Quiroga, 2017). Pero no se refiere a una autonomía pedagógica, sino de financiación.

El enfoque neoliberal maneja los términos descentralización y autonomía escolar de forma ambigua y calculada, para introducir tras estos significantes con carga positiva, significados radicalmente diferentes. Porque lo que las escuelas se ven obligadas a asumir, según la ideología neoliberal, es una 'autonomía' presupuestaria, en la que no vienen asegurados recursos económicos y humanos. 
La finalidad es presionar a cada escuela para que busque las vías de cara a limitar sus gastos cada vez más, en un contexto de radical recorte presupuestario, y a buscar otras fuentes de financiación privada y externa.

De hecho, durante la década de los noventa, las reformas educativas que se desarrollaron en EE.UU., Reino Unido, Canadá, Australia, Latinomérica, enfatizaban discursos que remarcaban términos de elección, responsabilidad, eficiencia y descentralización, ligados a una exigencia de mayor autonomía de funcionamiento de los centros educativos. El efecto aparentemente "paradójico" de estas reformas es que permitieron a los gobiernos centrales adquirir un control todavía mayor sobre la vida diaria de las instituciones escolares. Eso fue debido a la forma en que el proceso de descentralización se llevó a cabo: sin la asignación de los recursos necesarios. En la actualidad, tras casi treinta años de unas reformas que pretendían aumentar la autonomía educativa, las administraciones centrales han aumentado su poder a la hora de determinar las políticas escolares, los currículums y el proceso de evaluación. Incluso han creado instituciones dotadas con los medios para ejercitar un control más estrecho sobre el rendimiento de las escuelas. Lo más grave es que, ahora, quienes determinan el funcionamiento de los centros educativos no son los principios ni las necesidades educativas, sino los objetivos de los programas financieros de ajuste económico o programas de ajuste estructural (Fischman \& Gandin, 2008).

Ya no se trata de luchar por una mejora de la educación y el sistema educativo, de procurar más recursos donde más se necesiten, de dar más apoyos a aquellos centros con más dificultades, sino que se fomenta la autonomía, como forma de competencia entre centros educativos. Competencia que se ve acrecentada y potenciada por una política de exigencia de resultados. Unos en los que se concentra toda la población minoritaria o con necesidades, que están en zonas periféricas o rurales, con escasos recursos y profesorado interino lo cual impide la consolidación de un equipo estable, frente a otros que concentran a la población de clase media y alta, con muchos recursos. De esta forma aumentará aún más la brecha social que ya existe actualmente, consolidando la polarización social y destruyendo la igualdad de oportunidades y la cohesión social (Cañadell, 2015).

Esta competencia entre centros, auspiciada por las administraciones educativas con la publicación de rankings, potencia mecanismos de mercado donde las escuelas luchan para mostrarse o venderse como 'mejores' que las otras, para resaltar su supuesto "valor añadido", sea en términos de proyectos de bilingüismo, coaching o emprendimiento, instalaciones y recursos, resultados académicos, modelos disciplinarios, "innovaciones" de moda, etc.

Y las familias luchan para acceder a aquellas escuelas que aparecen en este mercado de oportunidades como las "mejores", las que están en los puestos superiores de los rankings. "Porque efectivamente las familias (sobre todo aquellas con más capital cultural) saben que no escogen entre escuelas diferentes sino entre escuelas eminentemente desiguales. Desiguales en oportunidades y condiciones para el aprendizaje y el desarrollo integral de sus hijos e hijas. $Y$ abi es donde radica la trampa de numerosos discursos. En tratar como sinónimos 'diferencia'y 'desigualdad" (Tarabini, 2017). Unas escuelas son gratuitas y otras cobran cuotas, que dificultan el acceso a determinados sectores sociales; unas concentran toda la diversidad de alumnado, que llega durante el curso por cuestiones migratorias y otras tienen un alumnado prácticamente "idéntico" durante toda la etapa escolar; unas tienen pantallas digitales en todas las aulas y otras no tienen ni ordenadores; unas tienen un profesorado estable, mientras otras tienen una plantilla docente que no deja de cambiar año tras año. Por eso, como explica esta especialista, los discursos que enfatizan las virtudes de la competencia entre centros, la autonomía para reforzar la especificidad y, por tanto, la diferencia entre centros y la libre selección de centro educativo, olvidan que la educación es un bien común y un derecho que hemos de garantizar a todos los niños y niñas en condiciones de igualdad, no una ventaja competitiva individual que hay que aprovechar en un entorno competitivo.

Además, esta manera de concebir la autonomía y la descentralización supone el traspaso de responsabilidades y funciones, desde la Administración a cada centro y a su comunidad educativa en particular. Una vez establecida este tipo de autonomía, los resultados ya no son responsabilidad 
del sistema, ni de la Administración, sino del propio centro, o sea, de los docentes que trabajan en él. De hecho, detrás de estos conceptos se esconde una "privatización" encubierta de los centros públicos, en el sentido de que no forman ya parte de un "todo" que es el sistema educativo, sino que cada uno se convierte en una entidad individual que debe entrar a competir con los demás y debe además rentabilizar sus recursos (exactamente igual que si fuera una empresa).

Se extienden así los "contratos-programa" entre la Administración y cada centro particular, que individualizan la relación con la Administración y donde ésta condiciona el aumento de la financiación a la presentación de resultados cuantificables. Contratos-programa que se dotan en función de los resultados obtenidos. Esto no sólo desarticula los mecanismos unificados de negociación con los sindicatos de trabajadores y trabajadoras de la educación y flexibiliza las formas de contratación y las retribuciones salariales de los colectivos docentes, sino que exime al Estado de su obligación de suministrar los recursos necesarios y garantizar la calidad del servicio, y permite que la Administración se desentienda progresivamente de la financiación de los centros públicos, especialmente de los que más necesitan apoyo y recursos dejando en manos de los "gestores educativos" la responsabilidad de dar una educación de calidad. De esta forma, la responsabilidad del éxito o fracaso del funcionamiento de los centros se delega en las comunidades educativas (Apple, 2002; Cañadell, 2005, 2016).

Estas estrategias para eludir la responsabilidad de la Administración educativa, a quienes se ha encomendado el diseño, planificación y la gestión de la educación pública, suelen envolverse en el lenguaje de la descentralización administrativa, la apertura a la comunidad, y la participación de los sectores implicados. Sin embargo, al mismo tiempo que se proclama este supuesto proceso de autonomía, esta estrategia se ve compensada por un no menos poderoso proceso centralizador fundado en el desarrollo de programas nacionales de control y evaluaciones estandarizadas de los contenidos educativos (básicamente, pruebas de rendimiento aplicadas a la población estudiantil); el diseño centralizado de reformas curriculares que establecen los contenidos básicos de un "currículum nacional"; y, la implementación de programas nacionales de formación de profesorado que permitan la actualización de los docentes según el plan curricular establecido en la citada reforma, centrados en esos mismos contenidos (emprendimiento, bilingüismo, TICs, etc.).

En suma, la salida que el neoliberalismo encuentra a la crisis educativa es producto de la combinación de una doble lógica centralizadora y descentralizadora: centralización del control pedagógico a nivel curricular en los contenidos establecidos a nivel central, a nivel de la evaluación estandarizada del sistema y a nivel de formación de los docentes (Medeiros, 2015); y descentralización de los mecanismos de financiamiento del sistema y gestión de los centros.

\section{OTRA EVALUACIÓN Y GESTIÓN ES POSIBLE Y NECESARIA}

Hemos de partir de una constatación ya expuesta: los estudios (Merchán, 2012; Mons, 2009; Sharon \& Berliner, 2007; Stobart, 2010) muestran que, a pesar de los recursos invertidos en evaluaciones estandarizadas desde los 1990s, no hay mejoría consistente y/o significativa en los resultados escolares de los países en los campos o aspectos evaluados. No ha habido ninguna evidencia empírica, en estas casi tres décadas transcurridas, de que, efectivamente, se haya producido una mejora del rendimiento del alumnado ni de la práctica educativa, más bien al contrario. Concluyen que realmente nos dicen muy poco (poco más de lo que ya sabemos) sobre las diferencias de rendimiento y nada sobre lo que habría que hacer para mejorar los resultados. Pero, además, destacan los efectos perversos de la política de rendición de cuentas y de los "efectos colaterales" de las pruebas estandarizadas. Pero, lo que es aún peor, demuestran que las evaluaciones estandarizadas nos introducen en un circuito peligroso donde se miden los "resultados esperados" y ajustados a las expectativas que reclama un determinado sistema escolar marcado esencialmente por las notas de exámenes. 
Por lo que, como primera y básica medida deberíamos salir del régimen PISA y de las evaluaciones estandarizadas, porque como dice Gentili (2014) "PIS A simplifica lo que es complejo. PIS A jerarquiza lo que no tiene un orden. PIS A compara lo incomparable. PIS A silencia lo que la realidad amplifica. PIS A distrae lo que merece atención. PIS A consagra lo que es banal y trivializa lo que debería ser fundamental'. Y eso sólo exige voluntad política de querer hacerlo. Lo cual no significa rechazar las evaluaciones, sino repensar el modelo y al servicio de qué y de quién se realizan. Puesto que la evaluación es una parte constitutiva del proceso formativo y una herramienta para reconocer sus avances y dificultades, por lo que debe ser un componente fundamental de toda política pública democrática.

Debemos, por tanto, replantear la evaluación como un proceso integral orientado a producir información, contextualizada social y culturalmente, de cara a ayudar a los centros educativos y mejorar realmente los procesos de enseñanza y aprendizaje. De hecho, actualmente se están llevando a cabo otro tipo de evaluaciones mucho mejor enfocadas. Por poner un solo ejemplo, hay muchas más ventajas en las pruebas $\mathrm{UNESCO}^{4}$ que en el reduccionismo analítico de PISA, como analiza la especialista ecuatoriana Rosa María Torres (2016).

Podríamos decir que evaluar en educación es fundamentalmente un proceso continuo de aprendizaje conjunto, de compartir nuestras miradas para comprender, entre todos los componentes de la comunidad y del sistema educativo, lo que hacemos, cómo lo hacemos, para qué y para quién lo hacemos, y cómo podemos ayudar a mejorarlo conjuntamente. Eso es lo que pretende hacer cotidianamente el profesorado en los centros educativos, pensando en común cómo mejorar su práctica docente, cómo ayudar a su alumnado con mejores métodos, cómo involucrar en este proceso apasionante a la comunidad educativa y a los propios estudiantes (Wrigley, 2007). Convertir los centros en organizaciones que aprenden conjunta y democráticamente a hacer las cosas cada vez mejor. E implicar a la administración educativa y a sus responsables en este proceso conjunto donde también podamos evaluarles para ayudarles a mejorar en sus políticas educativas, que a veces andan demasiado descarriadas.

Por eso esta concepción global de la evaluación debe conllevar la necesidad de una doble y simultánea evaluación. Interna de cada centro, es decir, una autoevaluación contextualizada, periódica e integral, con la participación de todos los actores implicados (profesorado, alumnado, familias y administración), que sirva para mejorar los procesos de enseñanza y aprendizaje y que incluya el funcionamiento específico de sus órganos de gobierno y departamentos, así como la efectividad de sus diferentes planes de centro y proyectos curriculares de etapa. Y una evaluación externa, complemento de la interna y coordinada con ella, porque ésta inevitablemente tiene sus puntos ciegos, pero a través de consultores invitados, revisiones de iguales por colegas de otros centros educativos u observaciones de especialistas universitarios, inspectores de educación o familias, que desarrollen funciones de asesoramiento y apoyo al profesorado, al alumnado y a la comunidad educativa, tanto en su tarea diaria como en los procesos de autoevaluación.

Además, las Administraciones educativas deberán diseñar, contando con sus organismos de evaluación y también con los Servicios de Inspección Educativa, un plan de evaluación externa de los programas educativos aplicados por la propia Administración y del funcionamiento de la propia Administración educativa. El plan de evaluación y los resultados obtenidos han de ser públicos y deben ponerse a disposición de la comunidad educativa para su conocimiento y debate.

Es necesaria, por tanto, una evaluación integral (que analice todos los factores que intervienen) del sistema educativo, formativa (orientada a la mejora), democrática (participada y conocida por la comunidad educativa y coordinada por el profesorado, la dirección de los centros y la inspección), diversa (sobre la autonomía de los centros, sus proyectos educativos, metodologías y contexto socioeducativo, que requiere diversas modalidades de evaluación), justa (que no compare realidades diferentes entre sî) y rigurosa (que use los instrumentos técnicos adecuados), donde se valoren cada vez más distintos tipos de capacidades cognitivas, entre otras, relacionadas con la comprensión, la

${ }^{4}$ http://www.unesco.org/new/es/santiago/education/education-assessment/ 
interpretación, la emoción, la convivencia, la participación, el análisis crítico, la democracia y el desarrollo del pensamiento creativo.

Así enfocada la evaluación, puede convertirse en un instrumento útil para todos los sectores implicados en la realidad educativa: para el alumnado (dándoles a conocer sus progresos y limitaciones, qué aspectos han planteado adecuadamente y cuáles han enfocado de forma incorrecta, qué alternativas y orientaciones tienen para subsanar las deficiencias; así como motivándoles en sus aciertos y ayudándoles en sus errores, utilizando la pedagogía del error como fuente de aprendizaje y de mejora constante); para el profesorado (informándoles sobre la adecuación de sus propuestas didácticas a las necesidades y características de su alumnado y sirviéndoles de base para la planificación e innovación y mejora de su diseño curricular); para las familias (a fin de darles información relevante que les permita cooperar y coordinar con la escuela en la tarea educativa); para el sistema educativo (de cara a ver igualmente sus insuficiencias y corregir y mejorar las políticas educativas llevadas a cabo).

En conclusión, la evaluación debe ser una evaluación democrática en la que participen como protagonistas las propias comunidades educativas. Debe permitir analizar los múltiples factores que inciden en ese proceso. Y debe ayudar a todos los actores que intervienen en él a mejorar las prácticas pedagógicas con un sentido formativo y no culpabilizador. Lógicamente, también debe ayudar a diseñar políticas y estrategias orientadas a mejorar las políticas gubernamentales en todos los campos de actuación.

Pero también este cambio de paradigma supone, en coherencia, otra gestión radicalmente distinta a la que propone el modelo neoliberal. Debemos abandonar la demanda neoliberal de soluciones tecnocráticas a los problemas educativos y asumir que, por encima de todo, la educación es una cuestión política y democrática y también de valores (Collet \& Tort, 2016). Y la gestión y organización que requieren las instituciones educativas es esencialmente democrática y participativa, no empresarial. Hemos de ser conscientes de que "la mejor democracia que se aprende en la escuela es la que se vive hoy en ella. La democracia y la participación se aprenden practicándolas, ejercitándolas y poniendo a prueba los límites y dificultades de las mismas" (Feu et al., 2016, 109). Si queremos una sociedad democrática, necesitamos escuelas democráticas, donde los equipos directivos asumen un liderazgo motivador y participativo que involucra a la comunidad educativa, promoviendo el sentimiento de comunidad que aprende. Donde la autonomía esté ligada a la posibilidad de innovación pedagógica de cada centro, a la adaptación del proceso de aprendizaje y enseñanza a los ritmos diferentes del alumnado específico de cada centro concreto; a un instrumento, en manos del profesorado y de la comunidad educativa de cada centro, para poder aplicar las estrategias pedagógicas y organizativas más convenientes y adaptadas a la diversidad del alumnado (Aróstegui \& Martínez, 2008). Este es el reto y la urgencia. 


\section{BIBLIOGRAFÍA}

Amigot, P. \& Martinez, L. (2015). Procesos de subjetivación en el contexto neoliberal. El caso de la evaluación del profesorado y la investigación universitaria. Revista de la Asociación de Sociología de la Educación, 8 (2), 138-155.

Andrews, P. \& al. (2014). OECD and Pisa tests are damaging education worldwide - academics. https://goo.gl/w8tdJw.

Apple, M. (2002). Educar "como Dios manda”. Mercados, niveles, religión y desigualdad. Barcelona: Paidós.

Aróstegui Plaza, J.L. \& Martínez Rodríguez, J.B. (Coords.). (2008). Globalización, posmodernidady educación: la calidad como coartada neoliberal. Madrid: Akal.

Ball, S.J. (2003b). The teacher's soul and the terrors of performativity. Journal of Education Policy, 18(2), 215-228.

Ball, S.J. (2003a) Profesionalismo, gerencialismo y performatividad. Educación y pedagogía, 37(15), 87-104.

Ball, S. J. (2016). Gobernanza neoliberal y democracia patológica. En J. Collet \& A. Tort. (Coord.). La Gobernanza Escolar (23-40). Madrid: Morata.

Bodin, A. (2007). What Does PISA Really Assess? What Does It Not? A French View. En G. Brinek, S. Hopmann \& M. Retzl (Eds.). PIS A According to PIS A - Does PIS A Keep What It Promises? (21-55). New Brunswick: Transaction Publishers.

Bolívar Botía, A. (2010). El liderazgo educativo y su papel en la mejora: Una revisión actual de sus posibilidades y limitaciones. Psicoperspectivas, (9), 2, 9-33.

Bolívar Botía, A. (2013). Cambio y liderazgo educativo en tiempos de crisis. Organización y gestión educativa: Revista del Fórum Europeo de Administradores de la Educación, (21), 4, 14-17.

Bolívar, A.; López Yáñez, J. \& Murillo, J. (2013). Liderazgo en las instituciones educativas. Una revisión de líneas de investigación. Fuentes, 14, 15-60.

Boltanski, L. (2009). De la critique. Précis de sociologie de l'émancipation. Paris: Gallimard.

Brinek, G. \& Hopmann, S. (2007). Introduction: PISA according to PISA. Does PISA Keep What It Promises? En G. Brinek, S. Hopmann \& M. Retzl (Eds.), PIS A According to PIS A - Does PIS A Keep What It Promise? (9-19). New Brunswick: Transaction Publishers.

Cañadell, R. (2005). El debate oculto sobre la educación. Cuadernos de Pedagogía, 346, 82-86.

Cañadell, R. (2016). La lucha educa. Movimientos sociales y renovación pedagógica: Educación, lucha y transformación social. Viento sur, 147, 47-55.

Carabaña Morales, J. (2015). La inutilidad de PIS A para las escuelas. Madrid: Catarata.

Carabaña Morales, J. (2016). El éxito de PISA y el poder de las clasificaciones. Con-Ciencia Social: Anuario de Didáctica de La Geografía, La Historia y Las Ciencias Sociales, (20), 157-163.

Collet, J. \& Tort, A. (Coord.). (2016). La Gobernanza Escolar. Madrid: Morata.

Deming, D. J., Cohodes, S., Jennings, J., \& Jencks, C. (2016). School accountability, postsecondary attainment, and earnings. Review of Economics and Statistics, 98(5), 848-862. DOI: https://doi.org/10.1162/REST_a_00598

Dizon-Ross, R. (2020). How does school accountability affect teachers? Evidence from New York City. Journal of Human Resources, 55(1), 76-118. DOI: 10.3368/jhr.55.1.1015.7438R1

Feldfeber, M. \& Assael, J. (2014). Declaración del Grupo de Trabajo CLACSO. Politicas educativas y derecho a la educación en América Latina y el Caribe. https://goo.gl/C1i2oa.

Feng, L., Figlio, D., \& Sass, T. (2018). School accountability and teacher mobility. Journal of Urban Economics, 103, 1-17. DOI: https://doi.org/10.1016/j.jue.2017.11.001

Fernández Cano, A. (2016). Una crítica metodológica a las evaluaciones PISA. Relieve: Revista ELectrónica de Investigación y EV aluación Educativa, 22(1). 
Fernández Enguita, M. (2018). Y si no te gusta, te aguantas. En torno a algunos indicadores del malestar del alumnado. En Fundación Europea Sociedad y Educación (Ed.), Indicadores comentados sobre el estado del sistema educativo español 2018 (pp. 150-153). Madrid: Fundación Ramón Areces.

Feu, J.; Simó, N.; Serra, C.; Canima, J. \& Lázaron, L. (2016). Elementos clave para una gobernanza democrática de la escuela: Dimensiones e indicadores. En J. Collet \& A. Tort. (Coord.). La Gobernanza Escolar (101-126). Madrid: Morata.

Fischman, G.E. \& Gandin, L.A. (2008). Escola Cidada y los discursos críticos de esperanza educativa. En P. McLaren \& J.L. Kincheloe (Eds.). Pedagogía crítica. De qué hablamos, dónde estamos (287-304). Barcelona: Graó.

Foucault, M. (1975). Vigilary Castigar. México: Ediciones Siglo XXI.

Foucault, M. (2006). Seguridad, territorio y población. Curso en el Collège de France 1977- 1978. Buenos Aires: Fondo de Cultura Económica.

Friz Echeverría, C. (2016). La universidad en disputa. Sujeto, educación y formación universitaria en la concepción neoliberal. Santiago de Chile, Ceibo Ediciones

García Garnica, M. (2016). Dirección pedagógica y liderazgo educativo: prácticas eficaces en centros públicos andaluces. Tesis doctoral. Granada: Universidad de Granada.

Gjefsen, H. M. \& Gunnes, T. (2016). The effects of school accountability on teacher mobility and teacher sorting. MPRA. https://cutt.ly/MamZDEd

Gentili, P. (1997). Cultura, política y currículo. Ensayos sobre la crisis de la escuela pública. Buenos Aires: Losada.

Gentili, P. (2017). Entrevista: "Para PISA, el buen docente es solo el que genera buenos resultados conforme a lo que esta prueba valora". Revista TE CCOO, 358, 22-26.

Gómez Jiménez, A. J. (2018). Desinforme Pisa. Publicaciones: Facultad de Educación y Humanidades Del Campus de Melilla, 48(2), 279-299.

González González, M.T. (2017). La dirección del centro escolar y el liderazgo pedagógico. Revista Padres y Maestros, 370, 6-11.

Grek, S. (2009). Governing by numbers: the PISA 'effect' in Europe. Journal of Education policy, 24(1), 23-37.

Guattari, F. \& Rolnik, S. (2006). Macropolítica. Cartografías del Deseo. Madrid: Traficantes de Sueños.

Hamilton, L. S., Stecher, B. M., \& Klein, S. P. (2002). Making sense of test-based accountability in education. New York: Rand Corporation.

Hardt, M. \& Negri, A. (2002). El Imperio. Barcelona: Paidós.

Hewitt, K. K. (2015). Educator evaluation policy that incorporates EVAAS value-added measures: Undermined intentions and exacerbated inequities. education policy analysis archives, 23, 76, 1-45.

Kirabo Jackson, C. (2012). Non-cognitive ability, test scores, and teacher quality: Evidence from 9 th grade teachers in North Carolina (No. w18624). National Bureau of Economic Research. DOI: $10.3386 / \mathrm{w} 18624$

Kirabo Jackson, C. (2018). What do test scores miss? The importance of teacher effects on nontest score outcomes. Journal of Political Economy, 126(5), 2072-2107. DOI: https://doi.org/10.1086/699018

Lane, S. (2014). Validity evidence based on testing consequences. Psicothema, 26(1), 127-135.

Laval, C. (2004). La escuela no es una empresa. El ataque neoliberal a la enseñanza pública. Barcelona: Paidós.

Laval, Ch. Y Dardot, P. (2013). La nueva razón del mundo. Ensayo sobre la sociedad neoliberal. Barcelona: Gedisa.

Leal De Barros, M., \& Perez Centeno, C. (2014). PISA en el contexto latinoamericano: los casos de Argentina, Brasil y Chile. Revista Latinoamericana de Educación Comparada, 5(6), 46-62. 
Lingard, B.; Sellar, S.; Hogan, A. \& Thompson, G. (2017). Commercialisation in Public Schooling (CIPS). New South Wales Teachers Federation: Sydney, NSW.

Medeiros Dos Santos, SH.D. (2015). Políticas de formación docente para la educación profesional: oposiciones y permanencias marcados por el ideario neoliberal. Acta Scientiarum. Education, 37(2), 165-175.

Merchán Iglesias, F. Javier. (2012). La introducción en España de la política educativa basada en la gestión empresarial de la escuela. Archivos Analiticos de Políticas Educativas. (20), 32, 1-27.

Mons, N. (2009). Effects théoriques et réels des politiques d'evaluation standardisée. Revue française de pédagogie, 169, 99-140.

Moreno-Hidalgo, M. \& Manso, J. (2017). La Nueva Gestión de lo Público (NGP) como tendencia educativa global y su impacto en la conformación de la identidad docente. RIESED Revista Internacional de Estudios sobre Sistemas Educativos (2), 7, 33-51.

Morton, K. (2017). DfE confirms participation in 'baby PISA' pilot. NurseryWorld. https://goo.gl/cW9qki

Mullis, I. V., Martin, M. O., \& Hooper, M. (2017). Measuring changing educational contexts in a changing world: Evolution of the TIMSS and PIRLS questionnaires. En Rosén M., Yang Hansen K., Wolff U. (Eds). Cognitive abilities and educational outcomes. Methodology of Educational Measurement and Assessment (pp. 207-222). Springer: Cham.

Navarro Weckmann, M.A.; Guzmán Ibarra, I. \& Guaderrama Martínez, X.R. (2016). Una mirada sobre la dirección escolar. En José Luis Bernal Agudo (Coord). Globalización y organizaciones educativas: Comunicaciones (323-330), Zaragoza: CIOIE.

OCDE (2013). Synergies for Better Learning: An International Perspective on Evaluation and Assessment. Paris: OECD Publishing.

OCDE (2017). The Funding of School Education: Connecting Resources and Learning. Paris: OECD Publishing.

Ong, A. (2007). Neoliberalism as a mobile technology. Transactions of the Institute of British Geographers, 32(1), 3-8.

Perrenoud, Ph. (1990). La construcción del éxito y el fracaso escolar. Madrid: Morata

Quiroga, A. R. (2017). Escuela y producción de subjetividad. El papel de la educación en las sociedades del gerenciamiento y el paradigma de la gestión escolar. Ixtli. Revista Latinoamericana de Filosofía de la Educación, 4(8), 221-235.

Ravitch, D. (2013). Reign of Error: The Hoax of the Privatization Movement and the Danger to America's Public Schools. New York: Knopf Doubleday.

Ritzer, G. (1993). The McDonaldization of Society: An Investigation into the Changing Character of Contemporary Social Life. Newbury Park, CA: Pine Forge Press.

Rose, N., O'malley, P. \& Valverde, M. (2006). Governmentality. Annual Review of Law and Social Sciences, 2, 83-104.

San Martín, A. (2006). La organización de los centros escolares al trasluz del tamiz digital. En Sancho Gil, J. Mª. (Coord.). Tecnologías para transformar la educación. Madrid: Universidad Internacional de Andalucía - Akal.

Sebastián Heredero, E.; Catalán Cueto, J.P.; Herrera, F.; Utrera Infantes, C.; Acosta Vargas, J.S. \& Martín Bris, M. (2016). El liderazgo en la dirección y gestión para instituciones educativas en España e Iberoamérica. En José Luis Bernal Agudo (Coord). Globalización y organizaciones educativas: Libro de Simposios (70-103), Zaragoza: CIOIE.

Sharon, N. \& Berliner, D. (2007). Collateral Damage. How Highstakes testing corrupts America's schools. Cambridge, Mass.: Harvard Education Press.

Stobart, G. (2010). Tiempos de pruebas: Los usos y abusos de la evaluación. Madrid: Morata.

Tarabini, a. (2017). De nuevo con la elección escolar: o cuando la diferencia se confunde con la desigualdad. El diario de la Educación. https://goo.gl/9o9bTq

Vaca Uribe, J. (2005). PISA sin prisa. Revista CPU-E, (1), 3. 
Vargas jiménez, I. (2017). Mirada de la comunidad educativa acerca del desempeño de directivas educativas: Algunas reflexiones. Revista Electrónica Educare, 21, 1, 1-7.

Verger, A.; Bonal, X. \& Zancajo, A. (2016). Recontextualización de políticas y (cuasi)mercados educativos. Un análisis de las dinámicas de demanda y oferta escolar en chile. Archivos Analiticos de Politicas Educativas, 24(27), 42-54.

Verger, A. \& Parcerisa, Ll. (2017). La globalización de la rendición de cuentas en el ámbito educativo: una revisión de factores y actores de difusión de políticas. RBPAE, 33 (3), 663684.

Wrigley, T. (2007). Escuelas para la esperanza. Una nueva agenda hacia la renovación. Madrid: Morata. 


\section{SOBRE EL AUTOR}

\section{Enrique Javier Díez Gutiérrez}

Profesor de la Facultad de Educación de la Universidad de León. Doctor en Ciencias de la Educación. Licenciado en Filosofía. Diplomado en Trabajo Social y Educación Social. Ha trabajado también como educador social, como maestro de primaria, como profesor de secundaria, como orientador en institutos y como responsable de atención a la diversidad en la administración educativa. Especialista en organización educativa, desarrolla su labor docente e investigadora en el campo de la educación intercultural, el género y la política educativa. Entre sus últimas publicaciones se encuentran: La Asignatura Pendiente (Plaza \& Valdés, 2020); La Educación en venta (Octaedro, 2020); Educación para el Bien Común. Hacia una práctica crítica, inclusiva y comprometida socialmente (Octaedro, 2020); La revuelta educativa neocon (Trea, 2019); Neoliberalismo educativo (Octaedro, 2018); La polis secuestrada: Propuestas para una ciudad educadora, con Juan Ramón Rodríguez (Trea, 2018); La educación que necesitamos, con Alberto Garzón (Akal, 2016); Qué hacemos con la Universidad, con Adoración Guamán y Josep Ferrer (Akal, 2014); Desvelando la historia, con Mary Nash (Comares, 2013).

Contact information: Universidad de León (España), Facultad de Educación, 146. Campus de Vegazana, s/n. 24071 Universidad de León. Teléfono. Correo electrónico: ejdieg@unileon.es 